\title{
Escala de Autoeficácia Ocupacional em Intervenções com Populações Vulneráveis
}

\author{
Clarissa Pinto Pizarro de Freitas ${ }^{1}$ \\ Universidade Salgado de Oliveira \\ Bruno Figueiredo Damásio \\ Universidade Federal do Rio de Janeiro \\ Silvia Helena Koller \\ Universidade Federal do Rio Grande do Sul \\ Optentia Research Focus Area - North-West University \\ Vanderbijlpark, South Africa
}

\begin{abstract}
RESUMO - Foram investigadas as evidências de validade da Escala de Autoeficácia Ocupacional em Intervenções com Populações Vulneráveis (EAO-IPV). A amostra foi composta por 549 profissionais da Rede de Proteção ( $88 \%$ mulheres), com idade média de 37,8 anos ( $\mathrm{DP}=10$ anos). A estrutura fatorial da escala foi investigada por meio de modelagem de equações estruturais exploratória (MEEE). Os resultados indicaram a solução unifatorial da versão de 18 itens como a mais adequada. Os resultados das análises de validade concorrente e convergente estiveram no sentido esperado. Os índices de autoeficácia ocupacional para intervenções com populações em vulnerabilidade estiveram positivamente associados aos níveis de autoeficácia ocupacional, aos afetos positivos e à realização profissional, e negativamente associados aos afetos negativos, exaustão emocional e despersonalização.
\end{abstract}

Palavras-chave: autoeficácia ocupacional, vulnerabilidade social, escala, evidências de validade

\section{Occupational Self-Efficacy in Interventions for Populations in Social Vulnerability Scale}

\begin{abstract}
The evidences of validity of Occupational Self-Efficacy in Interventions for Populations in Social Vulnerability Scale (OSE - IPSV - S) were evaluated. The sample included 549 professionals of Welfare Network ( $88 \%$ female), mean age 37.8 years $(\mathrm{SD}=10$ years). The factorial structure of the scale was investigated through an exploratory structural equation modeling (ESEM). Results pointed to a one-factor solution of the 18 items version as the most reliable to the data. The results of the concurrent and convergente validity were in the expected direction. The levels of occupational self-efficacy in interventions for populations in social vulnerability were positively related to occupational self-efficacy, positive affect and personal accomplishment, and negatively correlated with negative affect, emotional exhaustion and depersonalization.
\end{abstract}

Keywords: occupational self-efficacy, social vulnerability, scale, evidence of validity

A vulnerabilidade social pode ser identificada nos contextos em que um indivíduo, grupo, sistema familiar ou comunidade pode ser lesado ou ter o desenvolvimento de suas potencialidades prejudicadas pela não disponibilidade dos recursos necessários ao enfrentamento de situações desafiadoras (e.g., aprendizado de novas habilidades) ou adversas (e.g., vivenciar catástrofes naturais; Vignoli, 2001). Os recursos podem ser materiais, simbólicos, culturais ou sociais, os quais impactam a qualidade de vida das pessoas e a forma que elas manejam diferentes situações que vivenciam. Além disso, os recursos são elementos que colaboram para o indivíduo, grupo, sistema familiar ou comunidade criar estratégias funcionais para alcançar um desempenho satisfatório nas tarefas que lhe são demandadas (e.g., por instituições escolares e organizações) e serem bem-sucedidos na preservação ou aumento dos recursos disponíveis

1 Endereço para correspondência: Universidade Salgado de Oliveira, Rua Marechal Deodoro, 263/Mestrado em Psicologia, Centro - Niterói, RJ, Brasil. CEP: 24.030-060.E-mail: freitas.cpp@gmail.com
(Abramovay, Castro, Pinheiro, Lima, \& Martinelli, 2002; Vignoli, 2001).

Os grupos que vivenciam situações de vulnerabilidade social estão inseridos em um ambiente com escassez de recursos que contribuam ao desenvolvimento pleno de suas potencialidades. Esses indivíduos podem vivenciar desvantagens em seu desempenho e dificuldades de mobilidade social (Abramovay et al., 2002; Morais, Paludo, \& Koller, 2010; Vignoli, 2001). Em razão disso, as populações em situação de vulnerabilidade social podem apresentar maior probabilidade de serem expostos a situações adversas, tais como violência, impossibilidade de acesso a serviços de saúde e educação de qualidade, sendo afetados em maior intensidade por catástrofes naturais (Abramovay et al., 2002; Morais et al., 2010).

Nesse contexto de adversidades, os serviços da Rede de Proteção podem constituir-se como uma estratégia ao desenvolvimento de recursos simbólicos e materiais que contribuam para a melhoria das condições de vida do indivíduo, sua família e comunidade (Finkler, 2011; Vignoli, 
2001). Isso pode ser observado, tendo em vista que as ações desenvolvidas pelos profissionais da Rede de Proteção visam a potencializar os recursos sociais disponíveis, prevenir ações de violência, proteger as vítimas e promover o seu desenvolvimento saudável (Brasil, 2009).

No Brasil, existem serviços públicos especializados no desenvolvimento de políticas públicas de combate às situações de vulnerabilidade social. Entretanto, esses serviços não conseguem, ainda, lidar de maneira satisfatória com a demanda existente (Freitas \& Habigzang, 2013; Luna, Ferreira, \& Vieira, 2010; Vignoli, 2001). Os altos níveis de demanda e as condições de trabalho precárias dificultam a atuação dos profissionais e a possibilidade de eles desenvolverem as intervenções de forma efetiva (Finkler, 2011). A ausência de qualificação profissional necessária para desenvolver suas atividades laborais adequadamente (Coles, Dartnall, \& Astbury, 2013; Freitas \& Habigzang, 2013) e a falta de apoio necessário para lidar com demandas de trabalho emocionalmente exaustivas (Shapiro, Dorman, Burkey, \& Welker, 1999) também são fatores que impedem os profissionais de fornecerem atendimento adequado aos usuários dos serviços públicos.

Em relação à capacidade de os profissionais manejarem suas tarefas laborais, observa-se que a presença de recursos no trabalho, tais como apoio social, autonomia, afetos positivos, autoeficácia geral e ocupacional, podem ser elementos que contribuem para que os profissionais atendam suas demandas de forma efetiva (Freitas, Silva, Damásio, Koller, \& Teixeira, 2014; Salanova, Llorens, \& Schaufeli, 2011; Schaufeli \& Salanova, 2014). A presença desses recursos também pode constituir-se como um fator protetivo contra o desenvolvimento de burnout e a experiência de afetos negativos (Consiglio, Borgogni, Alessandri, \& Schaufeli, 2013; Freitas et al., 2014).

Evidências têm indicado que profissionais que apresentam altos níveis de autoeficácia geralmente adaptam-se melhor ao ambiente de trabalho e podem avaliar altos níveis de demanda de trabalho de forma positiva (Bandura, 2000; Consiglio et al., 2013). A autoeficácia refere-se às crenças que a pessoa possui sobre suas habilidades de planejar e persistir no desenvolvimento das ações que possibilitarão a ela alcançar seus objetivos e resultados almejados em diferentes tarefas e em diversas situações (Bandura, 1997; Chen, Gully, \& Eden, 2001). Em razão de determinar os investimentos que serão realizados, esse recurso pessoal também impacta os níveis de motivação e envolvimento dos indivíduos no desenvolvimento das atividades planejadas (Akhtar, Ghayas, \& Adil, 2013; Bandura, 1997; Xanthopoulou, Bakker, \& Fischbach, 2013).

Em razão do potencial motivador da autoeficácia, esta pode atuar como um elemento determinante no envolvimento do profissional com as atividades que desenvolve em seu trabalho (Akhtar et al., 2013; Bandura, 1997; Xanthopoulou et al., 2013). A autoeficácia pode auxiliar os profissionais no enfrentamento de suas demandas e moderar as relações das condições de trabalho e dos níveis de bem-estar (Williams, Wissing, Rothmann, \& Temane, 2010; Xanthopoulou et al., 2013). Esta também está positivamente relacionada aos níveis de satisfação no trabalho e desempenho organizacional, estando negativamente relacionada aos índices de absenteísmo e rotatividade (Judge, Jackson, Shaw, Scott, \& Rich, 2007).

As dificuldades enfrentadas no desenvolvimento de políticas públicas de combate à vulnerabilidade social (Vignoli, 2001), associadas às condições de trabalho precárias às quais os profissionais da Rede de Proteção estão expostos (Finkler, 2011) indicam a relevância desses trabalhadores se considerarem capazes de atenderem suas demandas de trabalho (Ellet, 2009). Profissionais que se percebem capazes de realizar intervenções efetivas com populações em situação de vulnerabilidade apresentam maiores chances de serem bem-sucedidos no atendimento dessa população. Isso se deve ao fato de que, em razão dos altos níveis de autoeficácia ocupacional, esses profissionais provavelmente irão persistir mais no desenvolvimento de suas intervenções, assim como planejá-las de forma mais efetiva, em comparação aos seus colegas com baixos índices de autoeficácia ocupacional (Ellet, 2009).

Segundo Salanova, Peiró e Schaufeli (2002) a autoeficácia, enquanto um recurso profissional, deve ser avaliada de forma específica ao seu contexto, a fim de possibilitar que a relação desse fator com as outras dimensões presentes no trabalho seja compreendida em profundidade. Em razão disso, o presente estudo objetiva avaliar as evidências de validade de uma escala desenvolvida para investigar os níveis de autoeficácia ocupacional no desenvolvimento de intervenções com populações em situação de vulnerabilidade dos profissionais da Rede de Proteção.

\section{Método}

\section{Participantes}

A amostra foi composta por 549 profissionais da Rede de Proteção (88\% mulheres), com idade média de 37,8 anos $(D P=10$ anos). Os participantes trabalhavam em instituições de atendimento a populações em situação de vulnerabilidade (e.g., escolas, hospitais, Centros de Referência de Assistência Social, instituições de acolhimento) de seis regiões do Rio Grande do Sul, Brasil. O tempo de trabalho dos profissionais nas instituições variou de um mês a 36 anos $(M=6,6 ; M d=$ $41,0 ; M o=12,0 ; S D=6,7)$. O nível de escolaridade observado entre os profissionais foi: 15\% haviam concluído o Ensino Médio; $51 \%$ possuíam curso superior completo; e 34\% concluíram a pós-graduação.

\section{Instrumentos}

Escala de Autoeficácia Ocupacional em Intervenções com Populações Vulneráveis (EAO-IPV). A EAO-IPV objetiva investigar as crenças que os profissionais possuem sobre suas habilidades no que se refere às intervenções que possibilitem auxiliar populações em situação de vulnerabilidade. Especificamente, a EAO-IPV avalia aspectos relacionados à violação de direitos humanos, condutas de paz, e mediação de conflitos. Essa escala foi desenvolvida segundo as orientações propostas por Bandura 
(2000), nas quais sugere que a autoeficácia seja avaliada em um domínio específico, investigando o quanto o sujeito se sente capaz de lidar com diversas situações (situações essas que devem ser específicas ao que o pesquisador queira investigar). A EAO-IPV foi inicialmente composta por 27 itens, respondidos por meio de uma escala Likert de cinco pontos, variando de 1 (discordo totalmente) a 5 (concordo totalmente). Os itens foram construídos através de um amplo brainstorming (termo utilizado para se referir à troca de ideias, opiniões e informações sobre determinada temática) realizada pelos autores do presente estudo. A EAO-IPV foi desenvolvida com o objetivo de avaliar a autoeficácia frente a diversas situações comumente vivenciadas por profissionais que trabalham na rede de proteção a crianças e adolescentes em situação de vulnerabilidade social.

Escala de Autoeficácia Ocupacional (EAO-VR, Occupational Self-Efficacy Scale - Short Form - OSS-SF, Rigotti, Schyns, \& Mohr, 2008, adaptada por Damásio, Freitas, \& Koller, 2014). Avalia os níveis de autoeficácia ocupacional por meio de uma escala unidimensional. A escala é composta por seis itens, com alpha de Cronbach satisfatório ( $\alpha=0,90$; Rigotti et al., 2008). Na versão brasileira, apresentou uma estrutura unifatorial com adequada consistência interna $(\alpha=0,78)$. Os participantes respondem os itens segundo uma escala de cinco pontos, variando de 1 (discordo totalmente) a 5 (concordo totalmente). O alpha de Cronbach e os índices de ajuste do instrumento na presente amostra foram satisfatórios, sugerindo adequação da escala: $\alpha=0,80 ; C F I=0,97 ; T L I=0,94 ; \operatorname{RMSEA}(90 \% \mathrm{IC})=0,061$ $(0,036-0,089)$.

Escala de Bem-Estar Afetivo no Trabalho (JobRelated Affective Well-being Scale, JAWS, Van Katwyk, Fox, Spector, \& Kelloway, 2000, adaptada por Gouveia, Fonsêca, Lins, Lima, \& Gouveia, 2008). Investiga os afetos positivos e negativos vivenciados no trabalho. A versão original apresentou uma estrutura bifatorial, na qual cada escala utiliza 15 itens para investigar afetos positivos e 15 para avaliar os afetos negativos. Os índices de consistência interna satisfatórios (afetos positivos, $\alpha=0,94$; e afetos negativos, $\alpha=0,87$ ) foram satisfatórios. Neste estudo, foi aplicada a versão reduzida da JAWS (Gouveia et al., 2008), composta por 12 itens, dos quais seis avaliam afetos positivos e seis avaliam afetos negativos. Os itens são respondidos em uma escala de cinco pontos, variando de 1 (nunca) a 5 (sempre). A versão reduzida manteve a estrutura bifatorial do instrumento, sendo que os índices de consistência interna obtidos foram adequados (afetos positivos, $\alpha=0,78$; e afetos negativos, $\alpha=0,80)$. A consistência interna na presente amostra foi satisfatória (afetos positivos, $\alpha=0,86$; e afetos negativos, $\alpha=0,82$ ), assim como os índices de ajuste do instrumento, sugerindo adequação da escala: $C F I=0,98 ; T L I$ $=0,97$; RMSEA $(90 \% \mathrm{IC})=0,068(0,056-0,079)$.

Inventário Maslach de Burnout (Maslach Burnout Inventory, MBI, Maslach \& Jackson, 1981, adaptado por Lautert, 1995). Objetiva investigar os níveis de burnout por meio das dimensões exaustão emocional, despersonalização e realização pessoal. A consistência interna das três dimensões na versão original foi adequada (exaustão emocional, $\alpha=$ 0,90; despersonalização, $\alpha=0,77$; e realização pessoal, $\alpha$ = 0,74; Maslach \& Jackson, 1981). Na versão brasileira do
MBI, os coeficientes de $\alpha$ observados foram satisfatórios (exaustão emocional, $\alpha=0,86$; despersonalização, $\alpha=0,69$; e realização pessoal, $\alpha=0,76$; Lautert, 1995). As 22 questões são respondidas por meio de uma escala de cinco pontos, a qual varia de 1 (nunca) a 5 (sempre). A escala apresentou consistência interna adequada na amostra investigada (exaustão emocional, $\alpha=0,87$; despersonalização, $\alpha=$ 0,54 ; e realização pessoal, $\alpha=0,76$ ). Os índices de ajuste do instrumento na presente amostra foram satisfatórios, sugerindo adequação da escala: $C F I=0,93 ; T L I=0,92$; RMSEA (90\% IC) = 0,074 (0,069-0,079).

\section{Procedimento de Coleta de Dados}

O presente estudo constitui-se como um recorte da pesquisa Avaliação de Impacto de uma Capacitação Profissional para Trabalhadores da Rede de Proteção à Criança e ao Adolescente do Rio Grande do Sul, Brasil. Esse estudo objetivou promover a qualificação profissional e avaliar o impacto da capacitação sobre as intervenções desenvolvidas pelos profissionais no atendimento de populações em situação de vulnerabilidade. As temáticas trabalhadas foram: direitos humanos, definição e consequências da violência intrafamiliar, intervenções desenvolvidas no atendimento das vítimas de ações de violência e mediação de conflitos. Essa capacitação foi desenvolvida em seis municípios do Rio Grande do Sul.

Os profissionais que concordaram em colaborar com o estudo receberam uma cópia do Termo de Consentimento Livre e Esclarecido, sendo explicado que sua participação era voluntária. A aplicação dos questionários foi realizada no primeiro módulo da capacitação, de forma coletiva, em auditórios e salas de aula onde a capacitação foi desenvolvida. Esse estudo foi iniciado após a aprovação do Comitê de Ética da Universidade Federal de Ciências da Saúde de Porto Alegre (UFCSPA).

\section{Análise dos Dados}

Inicialmente, a amostra deste estudo foi dividida em duas partes. Com os dados resultantes da primeira parte da amostra $\left(n_{1}=284\right)$, foi realizada uma modelagem de equações estruturais exploratória (MEEE; em inglês, exploratory structural equation modeling, ESEM; Asparouhov \& Múthen, 2009), que consiste em uma técnica de análise fatorial exploratória que permite a avaliação de índices de adequação de ajuste do modelo. O número de fatores a ser retido foi investigado por meio do método Hull (LorenzoSeva, Timmerman, \& Kiers, 2011), o qual indicou a estrutura unidimensional da escala. A adequação de ajuste da EAOIPV foi avaliada por meio dos seguintes índices: CFI, TLI e RMSEA. De acordo com as diretrizes utilizadas (Brown, 2006), os valores de CFI e TLI devem estar acima de 0,90. Valores de RMSEA devem estar abaixo de 0,08 , sendo que o limite superior do intervalo de confiança (90\%) não deve ser superior a 0,100 . Índices de modificação (IM) foram utilizados, também, para buscar fontes de problemas na especificação do modelo. Erros correlacionados com IM 
acima de 50,00 foram considerados prejudiciais para a estimação do modelo, sendo, portanto, inspecionados.

Posteriormente, com os dados referentes à segunda parte da amostra $(n 2=265)$, foi avaliada uma versão refinada da escala, utilizando, novamente a técnica da MEEE. A avaliação do modelo confirmatório utilizou os mesmos índices de ajuste da MEEE (CFI; TLI; RMSEA). Para ambas as MEEE, foi utilizado o método de estimação Weighted Least Squares Mean and Variance-Adjusted (WLSMV; Muthén \& Muthén, 2010). O WLSMV é um método de estimação para dados ordinais que não exige pressuposto de normalidade das variáveis.

Em seguida, foram avaliadas evidências de validade concorrente e convergente para a EAO-IPV utilizando toda a amostra $(n=549)$. A análise de validação concorrente e convergente foi realizada utilizando as correlações de Spearman's rho, em razão da não-normalidade dos dados. Foram utilizados os valores fatoriais das escalas EAOIPV, EAO-VR, JAWS e MBI. A análise concorrente foi desenvolvida com a EAO-VR, foi esperado que a EAO-IPV apresentasse uma relação positiva e de magnitude moderada com o fator de autoeficácia ocupacional da EAO-VR. As hipóteses da análise convergente testadas foram que a autoeficácia ocupacional para intervenção com populações em situação de vulnerabilidade estaria positivamente associada aos afetos positivos do JAWS e à realização pessoal do MBI, e negativamente aos afetos negativos do JAWS, assim como às dimensões exaustão emocional e despersonalização avaliadas por meio do MBI.

\section{Resultados}

A dimensionalidade da EAO-IPV foi avaliada utilizando a amostra $1\left(n_{1}=284\right)$. O método Hull demonstrou que uma estrutura unidimensional seria a mais representativa para os dados. Assim, realizou-se a MEEE, sugerindo um fator a ser retido. Os resultados (ver tabela 1) indicaram que todos os itens carregaram adequadamente no fator estipulado. Entretanto, os índices de adequação de ajuste mostraram que o modelo unifatorial, constando os 27 itens, não poderia ser considerável aceitável. Com base nesses resultados, foi desenvolvido o refinamento da EAO-IPV. Para isso, duas estratégias foram adotadas. Primeiramente, estipulou-se como ponto de corte cargas fatoriais $>0,60$. Itens com cargas fatoriais abaixo de 0,60 (comunalidades $<$ de 36\%) foram excluídos da bateria. Assim, os itens 5, 8, 21 e 22 foram retirados do modelo.

Além disso, foi utilizado o critério dos índices de modificação (IM) para avaliar outras fontes de má especificação do modelo. Os IM permitem avaliar, entre outros aspectos, sobreposição de conteúdo entre os itens (Brown, 2006), o que é um conhecido fator de prejuízo aos modelos fatoriais confirmatórios (Byrne, 2010). Itens que apresentaram erros correlacionados com valores de IM acima de 50 foram inspecionados. Para cada par desses itens, optouse por excluir aquele que apresentava menor carga fatorial. Os pares de itens com IM acima de 50 foram: Itens 1-10 (IM =75,96; excluído o item 1; carga fatorial =0,608); Itens 2-3 $(\mathrm{IM}=55,54$; excluído o item 3 ; carga fatorial $=0,702)$; Itens
6-7 $(\mathrm{IM}=84,77$; excluído item 6; carga fatorial = 0,638); Itens 9-18 (IM = 90,96; excluído o item 9; carga fatorial = $0,730)$; Itens $14-15$ (IM = 77.67; excluído o item 15 ; carga fatorial $=0,706$ ). Ao final de todo o processo, foram excluídos os itens 1, 3, 5, 6, 8, 9, 15, 21 e 22, de modo que a EAO-IPV ficou com 18 itens.

Essa versão de 18 itens foi avaliada, por meio de uma nova MEEE, utilizando uma amostra independente (n2 $=265$ ). Os resultados (ver tabela 2) apresentaram uma versão mais parcimoniosa da escala, com índices de ajuste aceitáveis. Nessa amostra, nenhum par de itens apresentou IM acima de 20,0.

Após a estrutura unidimensional da EAO-IPV ser verificada, foram desenvolvidas análises concorrentes e convergentes com toda a amostra $(n=549)$. As análises descritivas (tendência central e desvio padrão) dos fatores são apresentadas na Tabela 3, assim como as correlações Spearman's rho entre os fatores.

\section{Discussão}

O presente estudo teve como objetivo investigar a estrutura fatorial da EAO-IPV. Para isso, foram realizadas duas MEEE com amostras independentes. A primeira MEE foi realizada com a escala original da EAO-IPV, a qual era composta por 27 itens, e a segunda com a versão refinada da escala, com 18 itens.

Os índices de adequação de ajuste obtidos na MEEE realizada com a EAO-IPV composta por 27 itens indicaram que esta solução não foi considera adequada. A fim de melhorar o ajuste da escala optou-se por retirar os itens que contribuíam menos do que $36 \%$ na explicação do fator $(5,8,21$ e 22). Além disso, foi observado que os seguintes pares de itens apresentavam sobreposição de conteúdo: 1 e 10 (enfrentamento de situações de violência); 2 e 3 (ações para proteção dos direitos dos indivíduos atendidos); 6 e 7 (difusão dos direitos humanos); 9 e 19 (atuação no combate à violência); 14 e 15 (atuação na promoção da igualdade; Brown, 2006). Foram excluídos os itens que apresentavam menor contribuição à explicação do fator (1, 3, 6, 9, e 15).

A segunda MEEE da EAO-IPV foi realizada com os 18 itens restantes. A versão refinada da EAO-IPV apresentou uma estrutura unifatorial, sendo que os 18 itens possuíam cargas fatoriais satisfatórias (entre 0,537 , item 4, a 0,781, item 10) e não foram observadas sobreposições de conteúdo nessa solução. A melhora nos índices de adequação de ajuste indica que o refinamento da EAO-IPV resultou no desenvolvimento de uma versão melhor da escala.

Esse processo de refinamento, além de promover a melhora dos índices de adequação de ajuste, possibilitou que os níveis de autoeficácia fossem acessados com uma escala breve e objetiva. O desenvolvimento de instrumentos breves para avaliar construtos, tal como a autoeficácia ocupacional, são de grande relevância às investigações realizadas no âmbito de psicologia organizacional e do trabalho. As vantagens de serem utilizados instrumentos breves confiáveis residem no fato de que estes evitam que ocorra a sobreposição entre os itens, reduzem as chances de 
Tabela 1. Modelagem de Equações Estruturais Exploratória da EAO-IPV $(n 1=284)$

\begin{tabular}{|c|c|}
\hline Itens (Conteúdo resumido) & Carga fatorial \\
\hline Item 1 (... violação de direitos humanos...) & 0,608 \\
\hline Item 2 (... agente de proteção aos direitos das pessoas...) & 0,713 \\
\hline Item 3 (... agir conforme as leis e diretrizes...) & 0,702 \\
\hline Item 4 (... pessoa tendo seus direitos violados...) & 0,735 \\
\hline Item 5 (... pensar sobre direitos humanos...) & 0,502 \\
\hline Item 6 (... respeito aos direitos humanos...) & 0,638 \\
\hline Item 7 (... preservação dos direitos humanos...) & 0,667 \\
\hline Item 8 (... identificar situações de violação de direitos humanos...) & 0,577 \\
\hline Item 9 (... falar sobre o tema "direitos humanos"...) & 0,730 \\
\hline Item 10 (... lidar com vários tipos de violência...) & 0,719 \\
\hline Item 11 (... identificar situações de violência contra as pessoas...) & 0,638 \\
\hline Item 12 (... pessoa está sendo maltratada eu consigo lhe ajudar...) & 0,666 \\
\hline Item 13 (... estratégias de prevenção a situações de violência...) & 0,686 \\
\hline Item 14 (... respeito à sua individualidade e a dos outros...) & 0,742 \\
\hline Item 15 (... companheirismo, ajuda e atenção nas relações humanas...) & 0,706 \\
\hline Item 16 (... prevenir ações de violência nas suas comunidades...) & 0,661 \\
\hline Item 17 (... valores e comportamentos que promovem a paz...) & 0,712 \\
\hline Item 18 (... falar sobre o tema "violência" em várias situações...) & 0,760 \\
\hline Item 19 (... lidar com diferentes conflitos que surgem nos grupos...) & 0,703 \\
\hline Item 20 (... agir adequadamente quando vejo pessoas discutindo...) & 0,710 \\
\hline Item 21 (... resolver conflitos sem o uso da agressividade...) & 0,517 \\
\hline Item 22 (... consigo trabalhar a noção de 'reconciliação'...) & 0,577 \\
\hline Item 23 (... identificar e antecipar situações que podem gerar conflitos...) & 0,628 \\
\hline Item 24 (... mediar situações de conflito sem interferir ou impor...) & 0,663 \\
\hline Item 25 (... mediar uma situação de conflito sem culpar os envolvidos...) & 0,645 \\
\hline Item 26 (... perceber interesses, sentimentos, percepções e vontades...) & 0,657 \\
\hline Item 27 (...auxiliar pessoas envolvidas em diferentes tipos de conflitos...) & 0,746 \\
\hline \multicolumn{2}{|l|}{ Índices de Adequação de ajuste do modelo } \\
\hline \multicolumn{2}{|c|}{ RMSEA $(90 \% \mathrm{CI})$} \\
\hline $0,116(0,11$ & 122) \\
\hline
\end{tabular}

os participantes deixarem questões em branco e permitem o desenvolvimento de um processo de coleta de dados efetivo.

A estrutura unifatorial observada na EAO-IPV foi considerada como a solução mais adequada. Essa estrutura unidimensional foi observada em diferentes instrumentos de mensuração de autoeficácia geral (Pacico, Hutz, \& Ferraz, 2014; Sbicigo, Teixeira, Dias, \& Dell'Aglio, 2012; Schwarzer \& Jerusalem, 1995) e ocupacional (Consiglio et al., 2013; Damásio et al., 2014; Rigotti et al., 2008; Salanova, Cifre, Martínez, \& Llorens, 2007). Esses achados demonstram que a estrutura unidimensional do construto de autoeficácia mantém-se quando são avaliados diferentes domínios da vida dos indivíduos.

Os níveis observados na EAO-IPV dos profissionais da Rede de Proteção estiveram fortemente associados aos índices de autoeficácia ocupacional. Esses fatores apresentaram uma variância compartilhada próxima a $50 \%$ $\left(r=0,68 ; r^{2}=46,2 \%\right)$. A relação de magnitude moderada entre as duas medidas de autoeficácia ocupacional evidencia que a EAO-IPV se constitui como um instrumento fidedigno. Além disso, pode ser observado que, apesar de investigarem o mesmo construto, os itens dos instrumentos não apresentam uma sobreposição de conteúdo. Isso ocorre porque a EAOVR foi desenvolvida para investigar os níveis autoeficácia no desenvolvimento de diferentes atividades laborais em diversas ocupações (Damásio et al., 2014; Rigotti et al., 2008). A EAO-IPV, por sua vez, investiga especificamente as crenças dos profissionais sobre suas habilidades em realizar intervenções com populações em situação de vulnerabilidade.

As correlações da EAO-IPV com as dimensões positivas relacionadas ao trabalho (afetos positivos e realização pessoal) foram positivas e moderadas. A EAO -IPV apresentou uma variância compartilhada de $9 \%\left(r^{2}\right)$ com os afetos positivos $(r=0,30)$ e de $25 \%\left(r^{2}\right)$ com a dimensão de realização profissional de burnout $(r=0,50)$. Essas relações eram esperadas, tendo em vista que a EAO-IPV constituise como um recurso no trabalho, o qual pode repercutir sobre as condições emocionais e afetivas vivenciadas pelos 
Tabela 2. Modelagem de Equações Estruturais Exploratória da EAO-IPV versão modificada (n2 = 265)

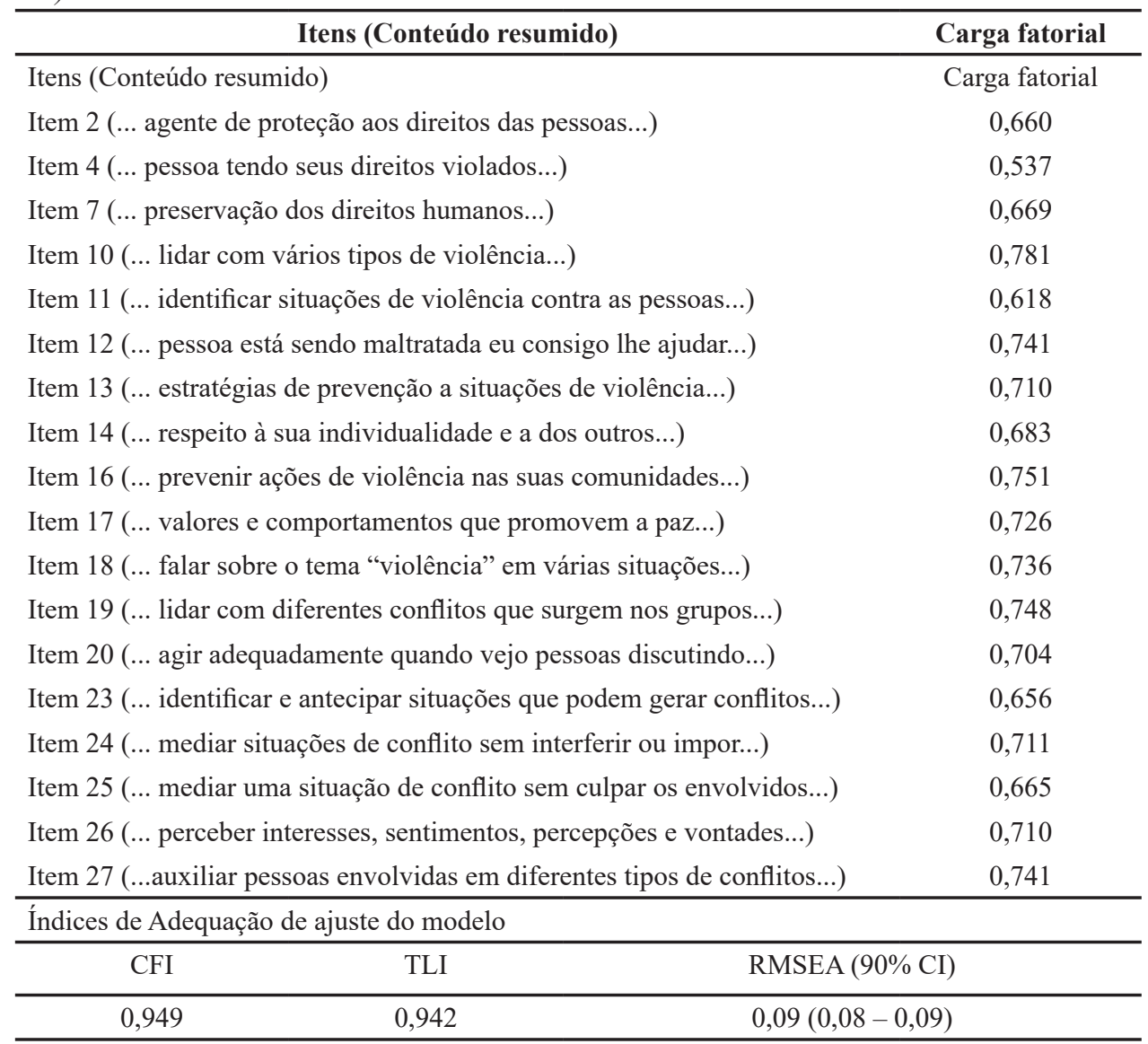

Tabela 3. Médias, Desvio-Padrão, e Correlações na Amostra $(n=549)$

\begin{tabular}{|c|c|c|c|c|c|c|c|}
\hline Dimensão & M (DP) & 1 & 2 & 3 & 4 & 5 & 6 \\
\hline 1. Autoeficácia Ocupacional em Intervenções com Populações Vulneráveis & $4,0(0,5)$ & & & & & & \\
\hline 2. Autoeficácia Ocupacional & $4,1(0,6)$ & $0,68^{*}$ & & & & & \\
\hline 3. Afetos Positivos & $4,2(0,6)$ & $0,30 *$ & $0,37 *$ & & & & \\
\hline 4. Afetos Negativos & $2,4(0,8)$ & $-0,20 *$ & $-0,30 *$ & $-0,56^{*}$ & & & \\
\hline 5. Exaustão Emocional & $2,1(0,6)$ & $-0,25^{*}$ & $-0,36^{*}$ & $-0,61 *$ & $0,65^{*}$ & & \\
\hline 6. Despersonalização & $1,6(0,5)$ & $-0,18^{*}$ & $-0,16^{*}$ & $-0,29^{*}$ & $0,32 *$ & $0,42 *$ & \\
\hline 7. Realização Pessoal & $4,1(0,4)$ & $0,50 *$ & $0,51 *$ & $0,57 *$ & $-0,40 *$ & $-0,51 *$ & $-0,30 *$ \\
\hline
\end{tabular}

profissionais (Consiglio et al., 2013; Guglielmi, Simbula, Schaufeli, \& Depolo, 2012).

As relações positivas entre os índices de autoeficácia ocupacional para intervenções com populações em vulnerabilidade com a realização profissional e os afetos positivos no trabalho também demonstram que a autoeficácia pode fortalecer a relação positiva do profissional com suas atividades laborais (Williams et al., 2010; Xanthopoulou et al., 2013). Essas relações evidenciam que a autoeficácia pode impactar as avaliações do profissional sobre as atividades laborais que eles desenvolvem, de modo a promover o aumento dos índices de motivação e bem-estar dos profissionais (Akhtar et al., 2013; Xanthopoulou et al., 2013).

Neste estudo, a magnitude da variância compartilhada entre a EAO-IPV e a realização profissional pode constituir-se como um indicador de fidedignidade da escala desenvolvida.
Esse entendimento origina-se da compreensão de que a realização profissional refere-se às avaliações dos profissionais sobre suas habilidades para manejar as demandas de trabalho (Demerouti \& Bakker, 2007; Salanova, Schaufeli, Llorens, Peiró, \& Grau, 2000).

Como esperado, as dimensões negativas do trabalho estiveram negativamente associadas à EAO-IVP. Foram observados baixos índices de variância compartilhada entre a EAO-IVP e os afetos negativos $\left(r=-0,20 ; r^{2}=4 \%\right)$, a exaustão emocional $\left(r=-0,25 ; r^{2}=6 \%\right)$ e a despersonalização $(r=$ 0,$\left.18 ; r^{2}=3 \%\right)$. Esses resultados corroboram a compreensão de que os recursos no trabalho apresentam relações de maior magnitude com os aspectos positivos (afetos positivos e realização pessoal), em comparação aos negativos (afetos negativos, exaustão emocional e despersonalização; Freitas 
et al., 2016; Salanova et al., 2011; Schaufeli \& van Rhenen, 2006).

As relações negativas entre os índices de autoeficácia ocupacional para intervenções com populações em vulnerabilidade com os níveis de exaustão emocional e despersonalização explicitam o fator protetivo da autoeficácia contra o desenvolvimento de burnout (Consiglio et al., 2013; Freitas et al., 2016). Similarmente, a autoeficácia ocupacional para intervenções com populações em vulnerabilidade esteve negativamente associada aos afetos negativos. Essas relações demonstram que a autoeficácia, além de atuar na promoção do bem-estar (Akhtar et al., 2013; Xanthopoulou et al., 2013), contribui para que os profissionais vivenciem afetos negativos em menor frequência (Consiglio et al., 2013; Freitas et al., 2016).

As limitações presentes nesta pesquisa referem-se aos instrumentos e às características da amostra investigada. $\mathrm{O}$ uso exclusivo de instrumentos de autorrelato pode ter produzido um viés relacionado ao método de coleta, sugerindo que os dados sejam analisados com cautela. Pesquisas futuras podem buscar testar a convergência da escala EAO-IPV correlacionando os índices de autoeficácia com indicadores externos, tais como avaliações de desempenho dos profissionais realizadas pelos respectivos supervisores e/ou colegas de trabalho.

Outro ponto importante a ser ressaltado refere-se ao baixo índice de consistência interna do fator despersonalização da escala de burnout. Entretanto, embora a fidedignidade alpha tenha sido abaixo do esperado, os índices de ajuste do modelo, como um todo, foram satisfatórios, o que sugere que a MBI pode ser uma medida aceitável para avaliação do burnout. Futuros estudos devem melhor inspecionar o fator despersonalização da MBI, com vistas a torná-lo mais preciso. Outra limitação foi a amostra ter sido composta predominantemente por participantes do sexo feminino. Compreende-se que essa limitação foi amenizada, pois a amostra analisada incluiu profissionais de diferentes idades, níveis de formação e tempo de trabalho, os quais atuavam nas áreas da saúde, educação e assistência social do estado do Rio Grande do Sul, Brasil.

Observa-se que é necessário o desenvolvimento de outros estudos investigando as evidências de validade da EAO-IPV com amostras maiores e mais diversificadas. Essas pesquisas contribuirão ao desenvolvimento da escala como uma ferramenta para a avaliação das condições de trabalho dos profissionais que atendem demandas complexas relacionadas às populações em situação de vulnerabilidade social.

\section{Considerações Finais}

O presente artigo contribui ao apresentar evidências iniciais de validade da EAO-IPV. Os resultados indicam que essa escala pode se constituir como uma ferramenta útil à avaliação da autoeficácia ocupacional enquanto um recurso pessoal no trabalho. O uso da EAO-IPV pode ser vantajoso por permitir que as crenças dos profissionais sobre suas habilidades em atender demandas de trabalho complexas sejam avaliadas por meio de uma escala breve. Além disso, a avaliação dos níveis de autoeficácia de uma atividade específica expande a compreensão do papel desse recurso pessoal sobre os níveis de motivação e desempenho dos profissionais (Bandura, 1997). Dessa forma, o acesso aos níveis de autoeficácia ocupacional em intervenções com populações em vulnerabilidade social dos trabalhadores possibilita o desenvolvimento de intervenções efetivas à promoção da qualificação profissional e de melhorias das condições de trabalho.

\section{Referências}

Abramovay, M., Castro, M. G., Pinheiro, L. C, Lima, F. S., \& Martinelli, C. C. (2002). Juventude, violência e vulnerabilidade social na América Latina: Desafios para políticas públicas. Brasília: UNESCO.

Akhtar, S., Ghayas, S., \& Adil, A. (2013). Self-efficacy and optimism as predictors of organizational commitment among bank employees. International Journal of Research Studies in Psychology, 2(2), 33-42. doi: 10.5861/ijrsp.2012.131

Asparouhov, T., \& Muthén, B. (2009). Exploratory structural equation modeling. Structural Equation Modeling, 16(3), 397-438. doi:10.1080/10705510903008204

Bandura, A. (1997). Self-efficacy: The exercise of control. New York: Freeman.

Bandura, A. (2000). Cultivate self-efficacy for personal and organizational effectiveness. In E. A. Locke (Ed.), Handbook of principles of organization behavior (pp. 120-136). Oxford, UK: Blackwell.

Brasil. (2009). Orientações técnicas: Centro de Referência de Assistência Social - CRAS. Brasília: Ministério do Desenvolvimento Social e Combate à Fome.

Brown, T. A. (2006). Confirmatory factor analysis for applied research. New York: The Guilford Press.

Byrne, B. M. (2010). Structural equation modeling with AMOS: Basic concepts, applications, and programming (2nd ed.). New York: Routledge, Taylor \& Francis.

Chen, G., Gully, S. M., \& Eden, D. (2001). Validation of a New General Self-Efficacy Scale. Organizational Research Methods, 4(1), 62-83. doi:10.1177/109442810141004

Coles, J., Dartnall, E., \& Astbury, J. (2013). "Preventing the pain" when working with family and sexual violence in primary care. International Journal of Family Medicine, 13, 10-15. doi: 10.1155/2013/198578

Consiglio, C., Borgogni, L., Alessandri, G., \& Schaufeli, W. B. (2013). Does self-efficacy matter for burnout and sickness absenteeism? The mediating role of demands and resources at the individual and team levels, Work \& Stress: An International Journal of Work, Health \& Organisations, 27(1), 22-42. doi: 10.1080/02678373.2013.769325

Damásio, B. F., Freitas, C. P. P., \& Koller, S. H. (2014). Adaptation and validation of the Brazilian Version of the Occupational Self-Efficacy Scale - Short form (OSS-SF). Revista Brasileira de Orientação Profissional, 15(1), 65-74.

Demerouti, E., \& Bakker, A. B. (2007). The Oldenburg Burnout Inventory: A good alternative to measure burnout and engagement. In J. Halbesleben (Ed.), Stress and burnout in health care (pp. 65-78). Nova York: Nova Sciences. 
Ellett, A. J. (2009). Intentions to remain employed in child welfare: The role of human caring, self-efficacy beliefs, and professional organizational culture. Children and Youth Services Review, 31(1), 78-88. doi:10.1016/j.childyouth.2008.07.002

Finkler, L. (2011). Evaluation of a social project for children and adolescents living on the streets and their families (Unpublished doctoral dissertation). Postgraduate Program in Psychology, Federal University of Rio Grande do Sul, Porto Alegre.

Freitas, C. P. P., \& Habigzang, L. F. (2013). Percepções de psicólogos sobre a capacitação para intervenção com vítimas de violência sexual (Perceptions of psychologists on training for intervention with victims of sexual violence). Psicologia Clínica, 25(2), 215-230. doi: 10.1590/S010356652013000200013.

Freitas, C. P. P., Silva, C. S. C., Damásio, B. F., Koller, S. H., \& Teixeira, M. A. P. (2016). Impact of Job-Related Well-Being on the Relationship of Self-Efficacy With Burnout. Paidéia, 26(63), 45-52. doi: 10.1590/1982-43272663201606.

Gouveia, V. V., Fonsêca, P. N. da, Lins, S. L. B., Lima, A. V. de, \& Gouveia, R. S. V. (2008). Escala de bem-estar afetivo no trabalho (JAWS): Evidências de validade fatorial e consistência interna (Scale of Job-Related Affective Wellbeing Scale (JAWS): Evidence of factorial validity and internal consistency). Psicologia: Reflexão e Crítica, 21(3), 464-473. doi: 10.1590/S0102-79722008000300015

Guglielmi, D., Simbula, S., Schaufeli, W. B., \& Depolo, M. (2012). Self-efficacy and workaholism as initiators of the job demandsresources model. Career Development International, 17(4), 375-389. doi:10.1108/13620431211255842

Judge, T. A., Jackson, C. L., Shaw, J. C., Scott, B. A., \& Rich, B. L. (2007). Self-efficacy and work-related performance: The integral role of individual differences. Journal of Applied Psychology, 92(1), 107-127. doi:10.1037/0021-9010.92.1.107

Lautert, L. (1995). O desgaste profissional do enfermeiro:The professional burnout of nurses (Unpublished doctoral dissertation). Postgraduate Program in Pontifical University of Salamanca, Salamanca.

Lorenzo-Seva, U., Timmerman, M. E., \& Kiers, H. A. L. (2011). The Hull Method for Selecting the Number of Common Factors. Multivariate Behavioral Research, 46(2), 340-364. doi:10.1 080/00273171.2011.564527

Luna, G. L. M., Ferreira, R. C., \& Vieira, L. J. E. S. (2010). Notificação de maus-tratos em crianças e adolescentes por profissionais da Equipe Saúde da Família. Ciência e Saúde Coletiva, 15(2), 481-491. doi: 10.1590/S141381232010000200025

Maslach, C., \& Jackson, S. E. (1981). The measurement of experienced burnout. Journal of Occupational Behavior, 2, 99-113. doi: 10.1002/job.4030020205

Morais, N. A., Paludo, S., \& Koller, S. H.. (2010). A família de crianças e adolescentes em situação de rua. In N. A. de Morais, L. Neiva-Silva \& S. H. Koller (Eds.), Endereço Desconhecido: Crianças e adolescentes em situação de rua (pp. 307-321). São Paulo: Casa do Psicólogo.

Muthén, L K, \& Muthén, B. O. (2010). Mplus: Statistical analysis with latent variables. User's guide. Los Angeles: Muthén \& Muthén.
Pacico, J. C., Ferraz, S. B., \& Hutz, C. (2014). Autoeficácia - Yes we Can! In C. S. Hutz (Ed.), Avaliação em Psicologia Positiva (pp. 111-119). Porto Alegre: Artmed.

Rigotti, T., Schyns, B., \& Mohr, G. (2008). A short version of the Occupational Self-Efficacy Scale: Structural and construct validity across five countries. Journal of Career Assessment, 16(2), 238-255. doi:10.1177/1069072707305763

Salanova, M., Schaufeli, W. B., Llorens, S., Peiró, J. M. \& Grau, R. (2000). Desde el 'burnout' al 'engagement': i Una nueva perspectiva? (From burnout to 'engagement': a new perspective?) Revista de Psicología del Trabajo y las Organizaciones, 16(2), 117-134. Retrieved from http://www. redalyc.org/revista.oa

Salanova, M., Cifre, E., Martínez, I., \& Llorens, S. (2007). Caso a caso en la prevención de riesgos psicosociales. Metodología WONT para una organización saludable. Bilbao: Lettera Publicaciones.

Salanova, M., Llorens, S., \& Schaufeli, W. B. (2011). "Yes, I can, I feel good, and I just do it!" On gain cycles and spirals of efficacy beliefs, affect, and engagement. Applied Psychology, 60(2), 255-285. doi: 10.1111/j.1464-0597.2010.00435.x

Salanova, M., Peiró, J. M., \& Schaufeli, W. B. (2002). Self-efficacy specificity and burnout among information technology workers: An extension of the job demand-control model. European Journal of Work and Organizational Psychology, 11(1), 1-25. doi: 10.1080/13594320143000735

Sbicigo, J. B., Teixeira, M. A. P., Dias, A. C. G., \& Dell'Aglio, D. D. (2012). Propriedades Psicométricas da Escala de Autoeficácia Geral Percebida (EAGP). Psico, 43(2), 139-146. Recuperado de http://revistaseletronicas.pucrs.br/ojs/index. php/revistapsico/article/view/11691/8037

Schaufeli, W.B., \& Salanova, M. (2014). Burnout, boredom and engagement at the workplace. In M. Peeters, J. de Jonge \& T. Taris (Eds.), People at work: An introduction to contemporary work psychology (pp. 293-320). Chichester: Wiley-Blackwell.

Schaufeli, W. B., \& van Rhenen, W. (2006). Over de rol van positieve en negatieve emoties bij het welbevinden van managers: Een studie met de Job-related Affective Well-being Scale (JAWS) (On the role of positive and negative emotions in the well-being of managers: A study of the Job-related Affective Well-being Scale (JAWS). Gedrag en Organisatie, 19(4), 323-344. doi:1874/133708

Schwarzer, R., \& Jerusalem, M. (1995). Generalized Self-Efficacy Scale. In J. Weinman, S. Wright \& M. Johnston (Eds.). Measures in health psychology: A user's portfolio. Causal and control beliefs (pp. 35-37). Windsor, UK: Nfer-Nelson.

Shapiro, J. P., Dorman, R. L., Burkey, W. M., \& Welker, C. J. (1999). Predictors of job satisfaction and burnout in child abuse professionals: Coping, cognition, and victimization history. Journal of Child Sexual Abuse, 7(4), 23-42. doi: 10.1300/ J070v07n04_03

Van Katwyk, P. T., Fox, S., Spector, P. E., \& Kelloway, E. K. (2000). Using the Job-Related Affective Well-Being Scale (JAWS) to investigate affective responses to work stressors. Journal of Occupational Health Psychology, 5(2), 219-230. doi:10.1037//1076-8998.5.2.219

Vignoli, J. R. (2001). Vulnerabilidad y grupos vulnerables: un marco de referencia conceptual mirando a los jóvenes. Santiago de Chile: CEPAL. 
Williams, S.-A., Wissing, M. P., Rothmann, S., \& Temane, Q. M. (2010). Self-efficacy, Work, and Psychological Outcomes in a Public Service Context. Journal of Psychology in Africa, 1(20), 43-52. Recuperado de http://www.ianrothmann.com/ pub/Williams_article.pdf
Xanthopoulou, D., Bakker, A. B., \& Fischbach, A. (2013). Work engagement among employees facing emotional demands: The role of personal resources. Journal of Personnel Psychology, 12(2), 74-84. doi:10.1027/1866-5888/a000085

Anexo I

Escala de Autoeficácia Ocupacional em Intervenções com Populações em Vulnerabilidade Social (EAO-IPV)

Abaixo está listada uma série de afirmações que dizem respeito a quanto você se sente capaz de lidar com diversas situações no seu dia-a-dia de trabalho. Avalie o seu grau de confiança em si mesmo e marque com um X a opção que melhor reflete a sua resposta. Utilize por base a escala abaixo: ATENÇÃO use a escala a seguir

\begin{tabular}{|c|c|c|c|c|c|c|c|c|}
\hline $\begin{array}{l}\text { (1) } \\
\text { Discordo } \\
\text { totalmente }\end{array}$ & $\begin{array}{l}\text { Discordo um } \\
\text { pouco }\end{array}$ & $\begin{array}{l}\text { (3) } \\
\text { Nem discordo } \\
\text { nem concordo }\end{array}$ & $\begin{array}{l}\text { Concordo um } \\
\text { pouco }\end{array}$ & \multicolumn{5}{|c|}{$\begin{array}{l}\text { (5) } \\
\text { Concordo } \\
\text { totalmente }\end{array}$} \\
\hline \multirow{2}{*}{\multicolumn{4}{|c|}{$\begin{array}{l}\text { 1) Eu consigo ser um agente de proteção aos direitos das pessoas com as quais eu } \\
\text { trabalho. } \\
\text { 2) Quando eu vejo uma pessoa tendo seus direitos violados, consigo agir adequadamente. }\end{array}$}} & 1 & 2 & 3 & & \\
\hline & & & & 1 & 2 & 3 & 4 & 5 \\
\hline \multicolumn{4}{|c|}{ 3) Eu consigo difundir a importância da preservação dos direitos humanos. } & 1 & 2 & 3 & 4 & 5 \\
\hline \multicolumn{4}{|c|}{ 4) Eu me sinto preparado para lidar com vários tipos de violência. } & 1 & 2 & 3 & 4 & 5 \\
\hline \multicolumn{4}{|c|}{ 5) Eu consigo identificar situações de violência contra as pessoas. } & 1 & 2 & 3 & 4 & 5 \\
\hline \multicolumn{4}{|c|}{$\begin{array}{l}\text { 6) Quando uma pessoa está sendo maltratada (ameaçada, agredida, discriminada, etc.), } \\
\text { eu consigo lhe ajudar de alguma maneira. }\end{array}$} & 1 & 2 & 3 & 4 & 5 \\
\hline \multicolumn{4}{|c|}{ 7) Eu sou capaz de desenvolver estratégias de prevenção a situações de violência. } & 1 & 2 & 3 & 4 & 5 \\
\hline \multicolumn{4}{|c|}{$\begin{array}{l}\text { 8) Eu consigo provocar nas pessoas a necessidade de que desenvolvam respeito à sua } \\
\text { individualidade e a dos outros. }\end{array}$} & 1 & 2 & 3 & 4 & 5 \\
\hline \multicolumn{4}{|c|}{$\begin{array}{l}\text { 9) Eu sou capaz de conversar com as pessoas para prevenir ações de violência nas suas } \\
\text { comunidades. }\end{array}$} & 1 & 2 & 3 & 4 & 5 \\
\hline \multicolumn{4}{|c|}{$\begin{array}{l}\text { 10) Eu consigo desenvolver com as pessoas com as quais eu trabalho, valores e } \\
\text { comportamentos que promovem a paz (não-violência). }\end{array}$} & 1 & 2 & 3 & 4 & 5 \\
\hline \multirow{2}{*}{\multicolumn{4}{|c|}{$\begin{array}{l}\text { 11) Eu sou capaz de falar sobre o tema "violência" em várias situações. } \\
\text { 12) Eu me sinto capaz de lidar com diferentes conflitos que surgem nos grupos em que } \\
\text { trabalho (ou que coordeno). }\end{array}$}} & 1 & 2 & 3 & 4 & 5 \\
\hline & & & & 1 & 2 & 3 & & 5 \\
\hline \multicolumn{4}{|c|}{ 13) Eu consigo agir adequadamente quando vejo pessoas discutindo. } & 1 & 2 & 3 & 4 & 5 \\
\hline \multicolumn{4}{|c|}{ 14) Eu consigo identificar e antecipar situações que podem gerar conflitos. } & 1 & 2 & 3 & 4 & 5 \\
\hline \multicolumn{4}{|c|}{$\begin{array}{l}\text { 15) Eu consigo mediar situações de conflito sem interferir ou impor uma solução ao } \\
\text { problema. }\end{array}$} & 1 & 2 & 3 & & 5 \\
\hline \multicolumn{4}{|c|}{ 16) Eu consigo mediar uma situação de conflito sem culpar os envolvidos } & 1 & 2 & 3 & + & 5 \\
\hline \multicolumn{4}{|c|}{$\begin{array}{l}\text { 17) Em uma situação de conflito, eu consigo perceber interesses, sentimentos, } \\
\text { percepções e vontades envolvidas na disputa. }\end{array}$} & 1 & 2 & 3 & & 5 \\
\hline \multicolumn{4}{|c|}{$\begin{array}{l}\text { 18) Geralmente eu consigo auxiliar pessoas envolvidas em diferentes tipos de conflitos a } \\
\text { construírem um acordo. }\end{array}$} & 1 & 2 & 3 & & 5 \\
\hline
\end{tabular}

